\title{
Seletividade de produtos fitossanitários utilizados na cultura do pessegueiro nos estágios imaturos de Trichogramma atopovirilia Oatman \& Platner, 1983 (Hymenoptera: Trichogrammatidae)
}

\author{
Selectivity of pesticides used in peach production to immature stages of Trichogramma atopovirilia \\ Oatman \& Platner, 1983 (Hymenoptera: Trichogrammatidae)
}

\author{
Fabrizio Pinheiro Giolo ${ }^{\mathrm{I}^{*}}$ Anderson Dionei Grützmacher ${ }^{\mathrm{II}}$ Cristiane Gindri Manzoni $^{\mathrm{I}}$ \\ Sandro Daniel NörnbergI Wagner da Roza Härter ${ }^{\mathrm{III}}$ Rodolfo Vargas Castilhos ${ }^{\mathrm{III}}$
}

\section{RESUMO}

Neste trabalho foi avaliada em condições de laboratório a seletividade dos produtos fitossanitários ( $\mathrm{g}$ ou $m L$ produto comercial $100 L^{-1}$ ) abamectina (80), carbaril (360), deltametrina (40), dimetoato (120), enxofre (600), etofenproxi (150), fentiona (100), fenitrotiona (150), fosmete (200), glufosinato de amônio (2L ha $\left.\mathrm{a}^{-1}\right)$, óleo mineral (2000) $e$ triclorfom (300), utilizados na cultura do pessegueiro, em relação a diferentes estágios imaturos de desenvolvimento do parasitóide de ovos Trichogramma atopovirilia Oatman \& Platner, 1983. Os bioensaios consistiram na pulverização direta dos produtos fitossanitários sobre ovos de Anagasta kuehniella (Zeller, 1879), contendo, em seu interior, o parasitóide em distintos estágios de desenvolvimento. A porcentagem de emergência de adultos do parasitóide foi avaliada. Reduções na emergência (RE) de adultos em relação à testemunha foram calculadas e os produtos fitossanitários classificados de acordo com índices propostos pela IOBC/WPRS. Produtos fitossanitários à base de abamectina, deltametrina, dimetoato, enxofre, etofenproxi, fosmete, glufosinato de amônio e óleo mineral não apresentaram reduções na emergência e foram classificados como inócuos $(<30 \%$ RE). Inseticidas à base de carbaril, fenitrotiona e triclorfom reduziram a porcentagem de emergência de adultos em todos os estágios imaturos de desenvolvimento do parasitóide, sendo levemente nocivos (30$79 \% R E$ ) ou moderadamente nocivos (80-99\% RE), conforme o estágio de desenvolvimento, enquanto que fentiona reduziu apenas nos estágios de pré-pupa e pupa, sendo classificado como levemente nocivo (30-79\% RE) para estes estágios. A associação dos métodos químico e biológico, na cultura do pessegueiro, é viável para os produtos fitossanitários inócuos quando T. atopovirilia encontra-se nos estágios imaturos de desenvolvimento.

Palavras-chave: Insecta, toxicidade, parasitóide de ovos, agrotóxicos, Prunus persica $L$.

\begin{abstract}
The selectivity of the pesticides ( $g$ or $m L$ commercial product $100 L^{-1}$ ) abamectin (80), carbaryl (360), deltamethrin (40), dimethoate (120), ethofenprox (150), fenthion (100), fenitrothion (150), glufosinate ammonium (2L $\left.h a^{-1}\right)$, mineral oil (2000), phosmet (200), sulphur (600) and trichlorphon (300) used in peach orchards was evaluated under laboratory conditions, expounded in different immature stages of the egg parasitoid Trichogramma atopovirilia Oatman \& Platner, 1983. Bioassays were carried out by direct applied of treatments on eggs of Anagasta kuehniella (Zeller, 1879) with the immature stages egg-larva, pre-pupa and pupa of $\mathbf{T}$. atopovirilia. The percentage of emergence adult parasitoids was assessed. Reduction in the emergence adult compared with the control was measure and the pesticides were classified according to IOBC/WPRS. Abamectin, deltamethrin, dimethoate, ethofenprox, glufosinate ammonium, mineral oil, phosmet and sulphur were not affected and were harmless $(<30 \%)$. The insecticides carbaryl, fenitrothion and trichlorphon affected the emergence adult in all developmental stages and were slightly harmful (30-79\%) or moderately harmful (8099\%) according developmental stage. Fenthion affected to pre-pupa and pupa stages and was slightly harmful (30-79\%). The association between biological and chemical control, in peach orchards, is possible for the pesticides harmless and immature stages of T. atopovirilia.
\end{abstract}

Key words: Insecta, side-effects, egg parasitoids, Prunus persica $L$.

\section{INTRODUÇÃO}

Entre as espécies frutíferas de clima temperado cultivadas no Brasil, o pessegueiro é cultivado em uma área de aproximadamente 24 mil

IPrograma de Pós-graduação em Fitossanidade, Departamento de Fitossanidade, Faculdade de Agronomia Eliseu Maciel (FAEM), Universidade Federal de Pelotas (UFPel), Campus Universitário s/n, CP 354, 96010-900, Pelotas, RS, Brasil.

${ }^{I I}$ Departamento de Fitossanidade, FAEM, UFPel, Pelotas, RS, Brasil. E-mail: adgrutzm@ufpel.tche.br. Autor para correspondência.

IIICurso de Agronomia, FAEM, UFPel, Pelotas, RS, Brasil. 
hectares. O Estado do Rio Grande do Sul (RS) é o principal produtor, com $52 \%$ da produção nacional. No entanto, as regiões produtoras de pêssego do RS são caracterizadas pelo baixo rendimento em relação a outros estados brasileiros (IBGE, 2005), principalmente, devido às condições climáticas favoráveis ao estabelecimento de pragas (insetos, doenças e plantas daninhas), o que promove a intensificação na utilização de produtos fitossanitários pelos persicultores nestas condições. A grafolita Grapholita molesta (Busck, 1916) (Lepidoptera: Tortricidae), também conhecida como broca-dos-ponteiros ou mariposa oriental, destaca-se como um dos principais insetos-praga que ocasionam perdas significativas à cultura do pessegueiro no RS (BOTTON et al., 2001). Recentemente, outro tortricídeo, conhecido popularmente como lagarta-das-fruteiras, Argyrotaenia sphaleropa (Meyrick, 1909) (Lepidoptera: Tortricidae), foi constatado em pomares de pessegueiro do RS, ocasionando danos de 1,8 a 2,2\% nos frutos (BOTTON et al., 2003).

Uma alternativa a ser incorporada ao manejo integrado da grafolita e da lagarta-das-fruteiras seria a utilização de parasitóide de ovos do gênero Trichogramma. Dentre as espécies deste parasitóide, Trichogramma atopovirilia Oatman \& Platner, 1983 (Hymenoptera: Trichogrammatidae) está amplamente distribuída na América do Sul e demonstra potencial no controle de tortricídeos como Ecdytolopha aurantiana (Lima, 1927) (Lepidoptera: Tortricidae) (MOLINA et al., 2005) e $\boldsymbol{G}$ molesta (STEFANELLO JÚNIOR et al., 2002).

Uma das limitações na utilização de parasitóides de ovos no controle da grafolita e da lagarta-das-fruteiras é a impossibilidade dos persicultores abdicarem totalmente do controle químico dessas pragas que prejudicam economicamente a produção de pêssego. O conhecimento da suscetibilidade a produtos fitossanitários de distintos estágios de desenvolvimento do parasitóide são imprescindíveis, pois estes normalmente são menos suscetíveis aos produtos fitossanitários por se encontrarem no interior do ovo hospedeiro. A partir do conhecimento destas informações, épocas alternativas de liberação dos parasitóides poderiam ser estipuladas, evitando-se coincidir o momento em que os produtos são pulverizados na cultura com o estágio de vida mais suscetível.

A avaliação da seletividade de produtos fitossanitários recomendados na cultura do pessegueiro vem sendo objeto de estudo em relação a sua ação aos parasitóides. Até o momento estão disponíveis informações, para alguns dos principais produtos recomendados nesta cultura, para estágios adultos e imaturos de Trichogramma cacoeciae Marchal, 1927 (GRUTZMACHER et al., 2004); estágios adultos e imaturos de Trichogramma pretiosum Riley, 1879 (GIOLO et al., 2005, 2006) e adultos de $\boldsymbol{T}$. atopovirilia (GIOLO et al., 2007). Em alguns destes estudos (GRUTZMACHER et al., 2004; GIOLO et al., 2006), ficou demonstrado que, para a grande maioria dos produtos fitossanitários avaliados, seria viável a integração dos métodos de controle químico e biológico e o parasitóide em estágio imaturo de desenvolvimento. No entanto, diferenças interespecíficas também foram evidentes, sugerindo que os estudos de seletividade devem ser conduzidos de preferência, com a espécie a ser utilizada no programa de controle biológico.

Considerando o potencial de utilização de T. atopovirilia como agente biológico de supressão populacional de $\boldsymbol{G}$ molesta e A. spharelopa em pomares de pessegueiro, objetivou-se no presente estudo avaliar a seletividade de doze produtos fitossanitários, comumente utilizados no controle de pragas da cultura do pessegueiro, em relação a diferentes estágios imaturos de desenvolvimento de T. atopovirilia em condições de laboratório.

\section{MATERIAL E MÉTODOS}

Os experimentos foram conduzidos nos laboratórios de Biologia de Insetos, Controle Biológico e de Pesticidas e Drogas do Departamento de Fitossanidade, Faculdade de Agronomia "Eliseu Maciel”, Universidade Federal de Pelotas, em Capão do Leão, RS e seguiram a metodologia sugerida pela IOBC/WPRS para T. cacoeciae (HASSAN, 1992; HASSAN \& ABDELGADER, 2001), adaptada para a espécie T. atopovirilia.

O material biológico utilizado nos experimentos foi constituído por parasitóides de ovos da espécie T. atopovirilia, proveniente da Escola Superior de Agricultura “Luiz de Queiroz” (ESALQ/ USP), SP. Esses parasitóides deram origem a uma criação em laboratório, mantida em câmaras climatizadas sob temperatura de $25 \pm 1^{\circ} \mathrm{C}$, umidade relativa de $70 \pm 10 \%$ e fotofase de 14 horas e, multiplicada em ovos inviabilizados em lâmpada germicida (STEIN \& PARRA, 1987), do hospedeiro alternativo Anagasta kuehniella (Zeller, 1879) (Lepidoptera: Pyralidae), criado conforme metodologia descrita por PARRA (1997), utilizando-se dieta artificial composta de farinha de trigo (97\%) e levedo de cerveja (3\%).

Cartões contendo 60 círculos de um cm de diâmetro com aproximadamente $300 \pm 50$ ovos por círculo de, no máximo, 24 horas de idade do hospedeiro $\boldsymbol{A}$. 
kuehniella foram expostos por um dia ao parasitismo por T. atopovirilia. Após o parasitismo, as fêmeas do parasitóide foram descartadas e os cartões contendo ovos supostamente parasitados foram transferidos para cilindros de vidro e acondicionados em câmaras climatizadas sob mesmas condições da criação até os parasitóides atingirem os períodos de desenvolvimento de 24 horas (um dia), 72 horas (três dias) e 168 horas (sete dias), correspondendo, respectivamente, aos estágios de ovo-larva, pré-pupa e pupa. Estes períodos foram atribuídos, tendo como referência, informações disponíveis para T. pretiosum (CÔNSOLI et al., 1999a). Para cada estágio de desenvolvimento, foi utilizado um cartão contendo 60 círculos.

Os produtos fitossanitários utilizados nos experimentos estão registrados ou em fase de testes para o controle de pragas na cultura do pessegueiro (Tabela 1), sendo a maioria recomendada na Produção Integrada de Pêssego (PIP) (FACHINELLO et al., 2003). Como foram testados 12 produtos, fez-se necessária a realização de dois experimentos (Experimento 1 e Experimento 2), cada um com seis agrotóxicos (Tabela 2). Os círculos contendo os parasitóides em diferentes fases de desenvolvimento (ovo-larva, pré-pupa e pupa) foram diretamente pulverizados com pulverizadores manuais de $580 \mathrm{~mL}$, que proporcionaram um volume de calda variando de $1,75 \pm 0,25 \mathrm{mg} \mathrm{cm}^{-2}$. O volume aplicado foi controlado por meio da pesagem, em balança eletrônica de precisão, antes e após a pulverização dos tratamentos. O excesso de umidade produzido pela calda foi evaporada, deixando os ovos tratados por cerca de três horas à temperatura ambiente. Após este período, os círculos com ovos foram transferidos para recipientes de vidro (10cm de comprimento e $2,5 \mathrm{~cm}$ de diâmetro) vedados na parte superior com tecido, preso com elástico, permitindo a ventilação e evitando a fuga dos parasitóides após emergência.

A porcentagem de emergência foi avaliada mediante contagem do número de adultos do parasitóide contido no recipiente de vidro em relação ao número total de ovos parasitados contidos no círculo de um cm de diâmetro. Ovos parasitados por Trichogramma adquirem uma coloração escura característica, que permite a identificação da ocorrência do parasitismo (CÔNSOLI et al., 1999a).

Cada tratamento foi repetido oito vezes, sendo que cada círculo continha $300 \pm 50$ ovos parasitados, considerando uma repetição no delineamento inteiramente casualizado em esquema fatorial (12 produtos fitossanitários e três estágios imaturos do parasitóide). Os resultados obtidos foram testados quanto a normalidade pelo teste de ShapiroWilk por meio do procedimento UNIVARIATE. Atendida esta premissa, os resultados foram submetidos a análise da variação (ANOVA) e comparação de médias por Tukey, por meio do procedimento GLM, em nível de 5\% de probabilidade de erro. Todas as análises foram realizadas com auxílio do programa estatístico SAS (SAS Institute, 2002). Reduções na emergência dos adultos para os produtos fitossanitários testados foram comparadas com a

Tabela 1 - Produtos fitossanitários utilizados na cultura do pessegueiro e avaliados nos testes de seletividade aos estágios imaturos de Trichogramma atopovirilia.

\begin{tabular}{|c|c|c|c|c|c|c|}
\hline Ingrediente ativo & Nome comercial & Classe $^{1}$ & Grupo químico & $\mathrm{DC}^{2}$ & C.i.a. ${ }^{3}$ & C.f.c. ${ }^{4}$ \\
\hline Abamectina & Vertimec $18 \mathrm{CE}^{5}$ & $\mathrm{~A} / \mathrm{I}$ & Avermectinas & 80 & 0,014 & 0,080 \\
\hline Carbaril & Sevin 480 SC $^{5}$ & I & Carbamato & 360 & 0,173 & 0,360 \\
\hline Deltametrina & Decis 25 CE & I & Piretróide & 40 & 0,010 & 0,040 \\
\hline Dimetoato & Tiomet $400 \mathrm{CE}^{5}$ & I & Fosforado & 120 & 0,048 & 0,120 \\
\hline Enxofre & Kumulus DF ${ }^{5}$ & $\mathrm{~F} / \mathrm{A}$ & Inorgânico & 600 & 0,480 & 0,600 \\
\hline Etofenproxi & Trebon 100 SC & I & Piretróide & 150 & 0,015 & 0,150 \\
\hline Fentiona & Lebaycid 500 & I & Fosforado & 100 & 0,050 & 0,100 \\
\hline Fenitrotiona & Sumithion $500 \mathrm{CE}^{5}$ & I & Fosforado & 150 & 0,075 & 0,150 \\
\hline Fosmete & Imidan $500 \mathrm{WP}$ & I & Fosforado & 200 & 0,100 & 0,200 \\
\hline Glufosinato de amônio & Finale $^{5}$ & $\mathrm{H}$ & $\begin{array}{l}\text { Homoalanina } \\
\text { substituída }\end{array}$ & $2 *$ & 0,200 & 1,000 \\
\hline Óleo mineral & Assist $^{5}$ & $\mathrm{I} / \mathrm{A}$ & Hidrocarboneto & 2000 & 1,512 & 2,000 \\
\hline Triclorfom & Dipterex $500^{5}$ & I & Fosforado & 300 & 0,150 & 0,300 \\
\hline
\end{tabular}

${ }^{1} \mathrm{~A}=$ acaricida, $\mathrm{F}$ = fungicida, $\mathrm{H}$ = herbicida, $\mathrm{I}=$ inseticida.

${ }^{2} \mathrm{DC}=$ Dosagem da formulação comercial em g ou mL $100 \mathrm{~L}^{-1}$ ou $* \mathrm{~L} \mathrm{ha}^{-1}$.

${ }^{3}$ C.i.a. $=$ Concentração (\%) testada do ingrediente ativo na calda.

${ }^{4}$ C.f.c. $=$ Concentração $(\%)$ testada da formulação comercial na calda.

${ }^{5}$ Produtos fitossanitários recomendados na Produção Integrada de Pêssego (PIP), segundo FACHINELLO et al. (2003). 
Tabela 2 - Porcentagem média ( \pm EP) de emergência de adultos de Trichogramma atopovirilia quando ovos do hospedeiro Anagasta kuehniella foram pulverizados com produtos fitossanitários contendo o parasitóide em diferentes estágios de desenvolvimento Temperatura: $25 \pm 1^{\circ} \mathrm{C}$; UR: $70 \pm 10 \%$; fotofase: 14 horas.

\begin{tabular}{|c|c|c|c|}
\hline \multicolumn{2}{|c|}{---------------------Tratamentos---------------------- } & \multicolumn{2}{|c|}{------------Estágio de desenvolvimento ${ }^{1}-----------$} \\
\hline Experimento I & Ovo-larva ${ }^{\text {n.s. }}$ & Pré-pupa & Pupa ${ }^{\text {n.s. }}$ \\
\hline Testemunha ${ }^{\text {n.s. }}$ & $97,70 \pm 1,58$ & $91,61 \pm 1,74 \mathrm{ab}$ & $104,65 \pm 2,17$ \\
\hline Abamectina ${ }^{\mathrm{ns} .}$ & $97,05 \pm 3,21$ & $91,48 \pm 3,95 a b$ & $105,78 \pm 4,81$ \\
\hline Enxofre ${ }^{\text {ns. }}$ & $98,89 \pm 5,17$ & $93,44 \pm 4,13 \mathrm{ab}$ & $90,85 \pm 5,18$ \\
\hline Etofenproxi & $97,96 \pm 4,26 \mathrm{~A}$ & $72,74 \pm 7,44 \mathrm{~b} \mathrm{~B}$ & $107,16 \pm 2,88 \mathrm{~A}$ \\
\hline Fosmete ${ }^{\text {n.s. }}$ & $98,92 \pm 6,16$ & $96,70 \pm 1,78 \mathrm{a}$ & $102,83 \pm 1,28$ \\
\hline Glufosinato de amônio ${ }^{\text {ns. }}$ & $108,60 \pm 3,14$ & $101,63 \pm 2,77$ a & $100,64 \pm 6,71$ \\
\hline Óleo mineral ${ }^{\text {n.s. }}$ & $103,35 \pm 2,44$ & $97,90 \pm 2,00 \mathrm{a}$ & $98,24 \pm 3,40$ \\
\hline Experimento II & Ovo-larva & Pré-pupa & Pupa \\
\hline Testemunha ${ }^{\text {n.s. }}$ & $109,03 \pm 1,49$ a & $107,45 \pm 1,52$ a & $95,71 \pm 4,19$ a \\
\hline Carbaril ${ }^{\text {n.s. }}$ & $43,35 \pm 1,64 b$ & $22,97 \pm 2,57 \mathrm{c}$ & $43,41 \pm 2,95 b$ \\
\hline Deltametrina ${ }^{\text {n.s. }}$ & $109,15 \pm 5,52 \mathrm{a}$ & $101,79 \pm 5,13 \mathrm{a}$ & $99,79 \pm 8,65 \mathrm{a}$ \\
\hline Dimetoato $^{\text {n.s. }}$ & $109,70 \pm 2,70 \mathrm{a}$ & $102,55 \pm 3,12$ a & $100,45 \pm 3,47 \mathrm{a}$ \\
\hline Fenitrotiona ${ }^{\text {n.s. }}$ & $4,18 \pm 1,20 \mathrm{c}$ & $3,35 \pm 0,91 \mathrm{c}$ & $17,00 \pm 3,05 c$ \\
\hline Fentiona & $94,25 \pm 3,27$ a $A$ & $65,39 \pm 5,47 \mathrm{~b} \mathrm{~B}$ & $57,39 \pm 9,41 b$ B \\
\hline Triclorfom & $52,79 \pm 6,18$ b A & $11,79 \pm 5,34$ с В & $15,10 \pm 4,12$ с В \\
\hline
\end{tabular}

${ }^{1}$ Médias não acompanhadas por letras idênticas, minúsculas na coluna e maiúsculas na linha, diferem significativamente em nível de 5\% de probabilidade de erro pelo teste de Tukey.

n.s. = não-si gnificativo.

testemunha e corrigidas por ABBOTT (1925). Aos resultados também foram atribuídos os índices propostos pela IOBC/WPRS, baseados nas reduções na emergência (RE) dos adultos dos tratamentos com produtos fitossanitários. Com base nestas reduções na emergência de adultos (RE), os produtos testados (Tabela 1) foram classificados segundo a IOBC/WPRS em: 1- inócuo (<30\% RE); 2- levemente nocivo (30-79\% RE); 3- moderadamente nocivo (80-99\% RE) e 4- nocivo (>99\% RE).

\section{RESULTADOS E DISCUSSÃO}

Os resultados dos bioensaios de seletividade dos produtos fitossanitários, quando pulverizados sobre diferentes estágios de desenvolvimento do parasitóide, para a porcentagem de emergência e redução na emergência de adultos de T. atopovirilia, estão apresentados, respectivamente, nas tabelas 2 e 3.

No Experimento I não houve diferenças na porcentagem de emergência de adultos em nenhum dos estágios de desenvolvimento avaliados em relação à testemunha (Tabela 2). Por outro lado, no Experimento II, a porcentagem de emergência de adultos de $\boldsymbol{T}$. atopovirilia foi inferior para os inseticidas carbaril, fenitrotiona e triclorfom em todos os estágios de desenvolvimento avaliados. O inseticida fentiona apresentou redução na porcentagem de emergência de adultos apenas quando o produto foi aplicado nos estágios de pré-pupa e pupa. Os demais produtos fitossanitários avaliados no Experimento II, semelhante ao bioensaio anterior, também não afetaram a emergência do parasitóide (Tabela 2).

Quando compararam-se os estágios de desenvolvimento para o mesmo produto fitossanitário (Tabela 2), observou-se que, no Experimento I, apenas o inseticida etofenproxi apresentou diferença, sendo o estágio de pré-pupa mais suscetível. No Experimento II, diferenças entre estágios de desenvolvimento foram obtidas apenas para os inseticidas fentiona e triclorfom, em que ambos apresentaram valores inferiores para a emergência de adultos nos estágios de pré-pupa e pupa (Tabela 2).

Na tabela 3 estão apresentadas as reduções na emergência de adultos de T. atopovirilia, para os respectivos estágios de desenvolvimento avaliados e a aplicação da classificação proposta pela IOBC/WPRS para testes de seletividade com parasitóides. Dos doze produtos fitossanitários avaliados, oito $(66,7 \%)$ foram considerados inócuos (classe 1) para todos os estágios de desenvolvimento avaliados. Quando considerados cada estágio de desenvolvimento, nove (75,0\%) produtos fitossanitários foram inócuos para o estágio de ovo-larva e oito produtos fitossanitários (66,7\%) para ambos os estágios, de pré-pupa e pupa. Apenas no Experimento II observaram-se reduções na emergência de adultos do parasitóide acima de $30 \%$, que é o limite superior da classe 1 (inócuo). O inseticida carbaril foi 
Tabela 3 - Redução na emergência (\%) de adultos de Trichogramma atopovirilia quando ovos do hospedeiro Anagasta kuehniella foram pulverizados com produtos fitossanitários, contendo o parasitóide em diferentes estágios de desenvolvimento e classificação de seletividade da IOBC para estes produtos fitossanitários. Temperatura: $25 \pm 1^{\circ} \mathrm{C}$; UR: $70 \pm 10 \%$; fotofase: 14 horas.

\begin{tabular}{|c|c|c|c|c|c|c|}
\hline \multirow{3}{*}{ Tratamentos } & \multicolumn{6}{|c|}{ - } \\
\hline & \multicolumn{2}{|c|}{-----Ovo-larva----- } & \multicolumn{2}{|c|}{------Pré-pupa------ } & \multicolumn{2}{|c|}{--------Pupa-------- } \\
\hline & $\mathrm{RE}^{1}$ & Classe $^{2}$ & $\mathrm{RE}$ & Classe & $\mathrm{RE}$ & Classe \\
\hline \multicolumn{7}{|l|}{ Experimento I } \\
\hline Abamectina & 0,67 & 1 & 0,14 & 1 & 0,00 & 1 \\
\hline Enxofre & 0,00 & 1 & 0,00 & 1 & 13,19 & 1 \\
\hline Etofenproxi & 0,00 & 1 & 20,60 & 1 & 0,00 & 1 \\
\hline Fosmete & 0,00 & 1 & 0,00 & 1 & 1,74 & 1 \\
\hline Glufosinato de amônio & 0,00 & 1 & 0,00 & 1 & 3,83 & 1 \\
\hline $\begin{array}{l}\text { Óleo mineral } \\
\text { Experimento II }\end{array}$ & 0,00 & 1 & 0,00 & 1 & 6,13 & 1 \\
\hline Carbaril & 60,24 & 2 & 78,62 & 2 & 54,64 & 2 \\
\hline Deltametrina & 0,00 & 1 & 5,27 & 1 & 0,00 & 1 \\
\hline Dimetoato & 0,00 & 1 & 4,56 & 1 & 0,00 & 1 \\
\hline Fenitrotiona & 96,17 & 3 & 96,88 & 3 & 82,24 & 3 \\
\hline Fentiona & 13,56 & 1 & 39,14 & 2 & 40,04 & 2 \\
\hline Triclorfom & 51,58 & 2 & 89,03 & 3 & 84,22 & 3 \\
\hline
\end{tabular}

${ }^{1} \mathrm{RE}=$ Redução na emergência de adultos de Trichogramma comparado com a testemunha do experimento.

${ }^{2}$ Classes da IOBC/WPRS para teste de seletividade sobre os estágios imaturos de Trichogramma: 1=inócuo (<30\%), 2=levemente nocivo (30-79\%), 3=moderadamente nocivo (80-99\%), 4=nocivo (>99\%).

levemente nocivo (classe 2), enquanto que fenitrotiona foi moderadamente nocivo (classe 3 ) a todos os estágios de desenvolvimento do parasitóide. Fentiona foi inócuo (classe 1) ao estágio de ovo-larva e levemente nocivo (classe 2) aos estágios de pré-pupa e pupa e triclorfom foi levemente nocivo (classe 2) ao estágio de ovo-larva e moderadamente nocivo (classe 3) aos estágios de pré-pupa e pupa (Tabela 3).

Dos produtos fitossanitários avaliados neste estudo, apenas carbaril, fenitrotiona, fentiona e triclorfom apresentaram algum efeito deletério sobre a emergência de adultos de T. atopovirilia, sendo a grande maioria $(75,0 \%)$ do grupo dos fosforados. Por outro lado, outros fosforados, tais como fosmete e dimetoato, os piretróides deltametrina e etofenproxi, o herbicida glufosinato de amônio, e demais produtos fitossanitários, como abamectina, enxofre e óleo mineral, quando pulverizados sobre os estágios imaturos de ovo-larva, pré-pupa e pupa, não afetaram a emergência de adultos do parasitóide. Estes últimos, quando submetidos à classificação proposta pela IOBC/ WPRS, não apresentaram reduções acima de 30\%, ficando dentro da classe inócuo (classe 1).

Não foram encontrados na literatura resultados de bioensaios de seletividade de produtos fitossanitários sobre estágios imaturos da espécie $\boldsymbol{T}$. atopovirilia. No entanto, resultados similares para a porcentagem de emergência de adultos para Trichogramma spp. foram previamente relatados para óleo mineral, enxofre e triclorfom pulverizados sobre imaturos de T. cacoeciae (HASSAN, 1998; GRUTZMACHER et al., 2004) e para abamectina, deltametrina, etofenproxi e glufosinato de amônio para T. pretiosum (GIOLO et al., 2006). Por outro lado, a porcentagem de emergência de adultos de $\boldsymbol{T}$. atopovirilia obtida neste trabalho foi superior à observada em outros estudos para deltametrina (YOUSSEF et al., 2004), fentiona (GRUTZMACHER et al., 2004) e fosmete (HASSAN, 1998, STERK et al., 1999) sobre T. cacoeciae; para deltametrina (HOHMANN, 1991) e abamectina (CÔNSOLI et al., 1998; CARVALHO et al., 2001) com T. pretiosum e para etofenproxi com Trichogramma dendrolimi (Matsumura, 1925) (TAKADA et al., 2001).

Possíveis explicações para os distintos resultados obtidos podem estar relacionadas a características intrínsecas dos ovos hospedeiros, às espécies e/ou linhagens de Trichogramma, às concentrações dos produtos fitossanitários e às distintas metodologias utilizadas nos respectivos bioensaios. Com relação aos ovos do hospedeiro, características como permeabilidade do córion e das camadas adjacentes são variáveis de acordo com a espécie, a idade e o grau de desenvolvimento do ovo. Neste sentido, CÔNSOLI et al. (1999b) estudaram as estruturas de ovos de seis espécies de lepidópteros, incluindo A. kuehniella. Estes autores verificaram diferenças intraespecíficas na espessura do córion, especialmente do exocórion e na densidade protéica desta última camada, que provavelmente, seja um dos principais fatores responsáveis pela não penetração de determinados produtos fitossanitários observados neste estudo.

Ciência Rural, v.38, n.5, ago, 2008. 
A existência de diferenças de suscetibilidade interespecífica de Trichogramma spp. (BULL \& COLEMAN, 1985) e intraespecífica para T. pretiosum (CARVALHO et al., 2003a) a produtos fitossanitários já foram previamente relatadas e demonstram a necessidade de realização de estudos específicos com a espécie, se possível, a linhagem a ser utilizada no programa de controle biológico da cultura. Estas diferenças explicariam, em parte, a diferença entre os resultados, para alguns inseticidas que foram testados na mesma concentração do presente estudo (GRÜTZMACHER et al., 2004). Além disso, em alguns trabalhos (HASSAN, 1998; GRÜTZMACHER et al., 2004; YOUSSEF et al., 2004) foram utilizados ovos de Sitotroga cerealella (Olivier, 1819) (Lepidoptera: Gelechiidae) como hospedeiro, os quais, possivelmente, também contribuíram para a diferença entre os resultados. Em estudo conduzido por BULL \& COLEMAN (1985), ficou evidenciado que a concentração inseticida a qual os ovos parasitados foram expostos influencia significativamente a emergência de adultos. No presente estudo, fosmete, etofenproxi, abamectina e deltametrina foram pulverizados em concentrações inferiores àquelas utilizadas, respectivamente, por STERK et al. (1999), TAKADA et al. (2001), CARVALHO et al. (2003b) e YOUSSEF et al. (2004). Influências do hospedeiro e da espécie e/ou linhagem utilizada nos distintos estudos ainda poderiam ter influenciado os resultados obtidos e não podem ser descartadas.

Outro fator importante que deve ser destacado é a falta de padronização nos testes de seletividade de produtos fitossanitários a inimigos naturais (DEGRANDE et al., 2002), pois foram utilizadas distintas metodologias de bioensaios (TAKADA et al., 2001; CARVALHO et al., 2003b), o que também deve ser considerado.

A IOBC/WPRS preconiza que nenhum teste isolado pode estabelecer informação suficiente para avaliar os efeitos colaterais de produtos fitossanitários sobre organismos benéficos. Os resultados obtidos para os produtos fitossanitários carbaril, fentiona, fenitrotiona e triclorfom devem ser avaliados conjuntamente com os resultados dos demais testes propostos na seqüência de testes sugerida pela IOBC/ WPRS (HASSAN \& ABDELGADER, 2001), que incluem testes de persistência biológica, em casa-de-vegetação e testes a campo, em pomares de pessegueiro.

\section{CONCLUSÕES}

Os produtos fitossanitários (g ou $\mathrm{mL}$ produto comercial $100 \mathrm{~L}^{-1}$ ) à base de abamectina (80), deltametrina (40), dimetoato (120), enxofre (600), etofenproxi (150), fosmete (200), glufosinato de amônio (2L ha-1) e óleo mineral (2000) não apresentam reduções na emergência de adultos, em laboratório, e são classificados como inócuos a Trichogramma atopovirilia. Inseticidas à base de carbaril (360), fenitrotiona (150) e triclorfom (300) reduzem a porcentagem de emergência de adultos, em laboratório, em todos os estágios imaturos de desenvolvimento do parasitóide, sendo levemente nocivos ou moderadamente nocivos, conforme o estágio de desenvolvimento de T. atopovirilia, enquanto que fentiona (100) reduz a emergência de adultos apenas nos estágios de pré-pupa e pupa, sendo classificado como levemente nocivo para estes estágios.

\section{AGRADECIMENTOS E APRESENTAÇÃO}

Ao Conselho Nacional de Desenvolvimento Científico e Tecnológico (CNPq) e à Fundação de Amparo à Pesquisa do Estado do Rio Grande do Sul (FAPERGS), pelo suporte financeiro.

Parte da Tese de Doutorado apresentada pelo primeiro autor ao Programa de Pós-graduação em Fitossanidade da Universidade Federal de Pelotas (UFPel), Pelotas, RS, Brasil.

\section{REFERÊNCIAS}

ABBOTT, W.S. A method of computing the effectiveness of an insecticide. Journal of Economic Entomology, Lanham, v.18, n.1, p.265-267, 1925.

BOTTON, M. et al. Monitoramento da mariposa oriental Grapholita molesta (Busck, 1916) (Lepidoptera: Tortricidae) na cultura do pessegueiro. Bento Gonçalves: Embrapa CNPUV, 2001. 4p. (Embrapa-CNPUV. Comunicado Técnico, 38).

BOTTON, M. et al. Ocorrência de Argyrotaenia sphaleropa (Meyrick) (Lepidoptera: Tortricidae) danificando pêssegos na Serra Gaúcha, Rio Grande do Sul. Neotropical Entomology, Londrina, v.32, n.3, p.503-505, 2003.

BULL, D.L.; COLEMAN, R.J. Effects of pesticides on Trichogramma spp. Southwestern Entomologist, Dallas, v.8, p.156-168, 1985.

CARVALHO, G.A. et al. Seletividade de alguns produtos fitossanitários a duas linhagens de Trichogramma pretiosum Riley, 1879 (Hymenoptera: Trichogrammatidae). Ciência e Agrotecnologia, Lavras, v.25, n.3, p.583-591, 2001.

CARVALHO, G.A. et al. Bioatividade de produtos fitossanitários utilizados na cultura do tomateiro (Lycopersicum esculentum Mill.) a Trichogramma pretiosum Riley, 1879 (Hymenoptera: Trichogrammatidae) nas gerações $F$ e $F_{2}$. Ciência e Agrotecnologia, Lavras, v.27, n.2, p.261-270, 2003 a.

CARVALHO, G.A. et al. Side-effects of insecticides used in tomato fields on Trichogramma pretiosum (Hymenoptera, Trichogrammatidae). Acta Scientiarum, Maringá, v.25, n.2, p.275-279, 2003b. 
CÔNSOLI, F.L. et al. Side-effects of insecticides used in tomato fields on the egg parasitoid Trichogramma pretiosum Riley (Hym., Trichogrammatidae), a natural enemy of Tuta absoluta (Meyrick) (Lep., Gelechiidae). Journal of Applied Entomology, Berlin, v.122, n.1, p.43-47, 1998.

CÔNSOLI, F.L. et al. Developmental time and characteristics of the immature stages of Trichogramma galloi and T. pretiosum (Hymenoptera, Trichogrammatidae). Revista Brasileira de Entomologia, São Paulo, v.43, n.2, p.271-275, 1999a.

CÔNSOLI, F.L. et al. Ultrastructure of the natural and factitious host eggs of Trichogramma galloi and T. pretiosum Riley (Hymenoptera, Trichogrammatidae). International Journal of Insect Morphology and Embryology, Tucson, v.28, n.3, p.211-229, $1999 b$.

DEGRANDE, P.E. et al. Metodologia para avaliar o impacto de agrotóxicos sobre inimigos naturais. In: PARRA, J.R.P. et al. (Eds.). Controle biológico no Brasil. São Paulo: Manole, 2002. p.71-93.

FACHINELLO, J.C. et al. Normas técnicas e documentos de acompanhamento da produção integrada de pêssego. Pelotas: Universidade Federal de Pelotas. 2003. 92p. (Documentos 01).

GIOLO, F.P. et al. Seletividade de agrotóxicos indicados na produção integrada de pêssego a Trichogramma pretiosum Riley, 1879 (Hymenoptera: Trichogrammatidae). Revista Brasileira de Fruticultura, Jaboticabal, v.27, n.2, p.222-225, 2005.

GIOLO, F.P. et al. Toxicidade de agrotóxicos utilizados na cultura do pessegueiro para estágios imaturos de Trichogramma pretiosum Riley (Hymenoptera: Trichogrammatidae). BioAssay, Piracicaba, v.1, n.3, p.1-8, 2006.

GIOLO, F.P. et al. Toxicidade de agrotóxicos utilizados na cultura do pessegueiro sobre o parasitóide de ovos Trichogramma atopovirilia Oatman \& Platner, 1983 (Hymenoptera: Trichogrammatidae). Ciência Rural, Santa Maria, v.37, n.2, p.308-314, 2007.

GRÜTZMACHER, A.D. et al. The side-effects of pesticides used in integrated production of peaches in Brazil on the egg parasitoid Trichogramma cacoeciae Marchal (Hym., Trichogrammatidae). Journal of Applied Entomology, Berlin, v.128, n.6, p.377-383, 2004.

HASSAN, S.A. Guideline for the evaluation of side-effects of plant protection product on Trichogramma cacoeciae. IOBC/ WPRS Bulletin, Darmstadt, v.15, n.3, p.18-39, 1992.

HASSAN, S.A. The side-effects of 161 pesticides on the egg parasitoid Trichogramma cacoeciae Marchal (Hymenoptera: Trichogrammatidae). Egg Parasitoids, Cali, v.1, n.1, p.63-76, 1998.
HASSAN, S.A.; ABDELGADER, H. A sequential testing program to assess the effects of pesticides on Trichogramma cacoeciae Marchal (Hym., Trichogrammatidae). Bulletin IOBC/ WPRS, Darmstadt, v.24, n.4, p.71-81, 2001.

HOHMANN, C.L. Efeito de diferentes inseticidas sobre a emergência de Trichogramma pretiosum (Hymenoptera: Trichogrammatidae). Anais da Sociedade Entomológica do Brasil, Londrina, v.20, n.1, p.59-65, 1991.

IBGE . Produção agrícola municipal: culturas temporárias e permanentes - 2004. V.31, p.1-134, 2005. Capturado em 23 ago. 2006. Online. Disponível na Internet: http:// www.sidra.ibge.gov.br.

MOLINA, R.M.S. et al. Seleção de Trichogramma spp., para o controle de Ecdytolopha aurantiana, com base na biologia e exigências térmicas. Revista Brasileira de Entomologia, Curitiba, v.49, n.1, p.152-158, 2005.

PARRA, J.R.P. Técnicas de criação de Anagasta kuehniella, hospedeiro alternativo para produção de Trichogramma. In: PARRA, J.R.P.; ZUCCHI, R.A. (Eds.). Trichogramma e o controle biológico aplicado. Piracicaba: FEALQ, 1997. Cap.4, p.121-150.

SAS Institute. Getting started with the SAS learning edition. Cary, NC, 2002. 81p.

STEFANELLO JÚNIOR, G.J. et al. Parasitismo de espécies do gênero Trichogramma sobre ovos de Grapholita molesta (Busck, 1916) (Lepidoptera: Tortricidae) em condições de laboratório. In: CONGRESSO DE INICIAÇÃO CIENTÍFICA, 12., 2002, Pelotas-RS . Resumos... Pelotas-RS: UFPel, 2002. p.512. (CD-ROM - Ciências Agrárias).

STEIN, C.P.; PARRA, J.R.P. Uso da radiação ultravioleta para inviabilizar ovos de Anagasta kuehniella (Zeller, 1879) visando estudos com Trichogramma spp. Anais da Sociedade Entomológica do Brasil, Londrina, v.16, n.1, p.229-233, 1987.

STERK, G. et al. Results of the seventh joint pesticide testing programme carried out by the IOBC/WPRS-Working Group "Pesticides and Beneficial Organisms". BioControl, Netherlands, v.44, n.1, p.99-117, 1999.

TAKADA, Y. et al. Effects of various insecticides on the development of the egg parasitoid Trichogramma dendrolimi (Hymenoptera: Trichogrammatidae). Journal of Economic Entomology, Lanham, v.94, n.6, p.1340-1343, 2001.

YOUSSEF, A.I. et al. The side-effects of plant protection products used in olive cultivation on the hymenopterous egg parasitoid Trichogramma cacoeciae Marchal. Journal of Applied Entomology, Berlin, v.128, n.9, p.593-599, 2004. 